\title{
Construcción discursiva de significados en torno a las posibilidades del currículo escolar desde las voces de los estudiantes ${ }^{1}$
}

\author{
Construção discursiva de significados em torno das \\ possibilidades do currículo a partir das vozes dos alunos \\ Discursive Construction of Meanings around the \\ Possibilities of the Curriculum from the Voices of Students
}

\author{
Patricia Baeza-Duffy ${ }^{2}$ \\ Giselle Melo-Letelier ${ }^{3}$
}

\section{RESUMEN}

El objetivo de este estudio cualitativo consiste en analizar la construcción discursiva de significados en torno a las posibilidades del currículo desde las voces de los estudiantes. El análisis adopta una perspectiva que integra

1. Esta publicación está enmarcada en el Proyecto PIA CONICYT 160009 (actual ANID), de la Pontificia Universidad Católica de Valparaíso. Se agradece a esta universidad, a ANID (ex CONICYT), como así también a los colegios participantes en el proyecto por apoyar esta investigación.

2. Doctora en Lingüística. Centro de Investigación para la Educación Inclusiva, PIACONICYT CIE 160009. Pontificia Universidad Católica de Valparaíso. Valparaíso, Chile. https://orcid.org/0000-0002-1163-1241.E-mail: patricia.baeza@pucv.cl.

3. Profesora de Educación General Básica con mención en Matemáticas y Ciencias Naturales. Magíster@ en Didáctica de las Ciencias Experimentales. Escuela de Pedagogía y Centro de Investigación para la Educación Inclusiva, PIA-CONICYT CIE 160009. Pontificia Universidad Católica de Valparaíso. Valparaíso, Chile. https://orcid.org/00000003-2569-8743. E-mail: giselle.melo@pucv.cl. 
aportes del Modelo de la Valoración y las categorías de estrategias y representación de actores sociales tomadas de los Estudios Críticos del Discurso. El corpus está conformado por tres grupos focales en colegios públicos. En cuanto a los procedimientos de análisis, se consideran dos fases. Una primera fase descriptiva comprende un microanálisis de recursos con los que los estudiantes construyen su posicionamiento. En la segunda fase, se analiza la alineación y desalineación como prácticas sociales en relación con las posibilidades del curriculum escolar. Los resultados muestran que los estudiantes plantean sus propias propuestas y utilizan prosodias valorativas que funcionan como estrategias que legitiman las clases motivadoras, lúdicas, creativas, a cargo de expertos $y$ deslegitiman las clases pasivas y con inspectores poco afectuosos, desmotivados. Las estrategias de legitimación conforman macroestrategias de transformación del status quo. Los actores sociales son representados por medio de: activación, funcionalización y autorización.

Palabras clave: construcción discursiva de significados; posibilidades del currículo escolar; voces de los estudiantes; des/legitimación; representación de actores sociales.

\section{RESUMO}

O objetivo deste estudo qualitativo é analisar a construção discursiva de significados em torno das possibilidades do currículo a partir das vozes dos alunos. A análise adota uma perspectiva que integra as contribuições do Modelo de Avaliação e as categorias de estratégias e representação dos atores sociais extraídas dos Estudos Críticos do Discurso. O corpus é composto por três grupos focais em escolas públicas. Quanto aos procedimentos de análise, são consideradas duas fases. Uma primeira fase descritiva consiste em uma microanálise de recursos com os quais os alunos constroem seu posicionamento. Na segunda fase, o alinhamento e o desalinhamento são analisados como práticas sociais em relação às possibilidades do currículo escolar. Os resultados mostram que os alunos apresentam propostas próprias e utilizam prosódias avaliativas que funcionam como estratégias que legitimam aulas motivacionais, lúdicas, criativas, ministradas por especialistas e deslegitimam aulas com metodologias passivas e com inspetores pouco afetuosos e motivados. As estratégias de legitimação constituem macroestratégias de transformação do status. Os atores sociais são representados por meio de: ativação, funcionalização e autorização.

Palavras-chave: construção discursiva de significados; possibilidades do currículo escolar; vozes de estudantes; de legitimação; representação dos atores sociais. 


\section{ABSTRACT}

This qualitative study aims to analyze the discursive construction of meanings around the possibilities of the curriculum from the voices of the students. The analysis adopts a perspective that integrates the contributions from the Appraisal Model as well as the strategy categories and social actors representation taken from the Critical Discourse Studies. The corpus is made up of three focus groups in public schools. Regarding the analysis procedures, two phases are considered. A first descriptive phase comprises a microanalysis of resources with which the students build their perspective. In the second phase, alignment and misalignment are analyzed as social practices in relation to the possibilities of the school curriculum. Findings show that students propose their own suggestions of changes and use evaluative prosodies. They work to legitimize motivational, playful, creative classes, in charge of experts and to delegitimize classes with passive methodologies and with little affectionate and motivated inspectors. The legitimation strategies make up macrostrategies of transformation of the status. The social actors are represented by means of: activation, functionalization and authorization.

Keywords: discursive construction of meanings; possibilities of the school curriculum; student voices; del legitimization; representation of social actors.

\section{Introducción}

Las escuelas públicas chilenas están en una constante tensión entre el modelo educativo de mercado (López et al., 2018) y la necesaria demanda de la sociedad de resistir a la individualización de la enseñanza (Grinberg, 2016). En el centro de este dilema están los sujetos que aprenden- las y los estudiantes, ya sean niñas, niños o jóvenes- pidiendo a las y los profesores que den sentido a lo que aprenden (Grinberg, 2016). A esta tensión se suman las ideas adultocéntricas en las escuelas, ya que las y los estudiantes han sido considerados en un lugar subordinado al de los adultos (Vergara et al., 2015), cuya perspectiva muchas veces invisibiliza a los alumnos, sin confiar en las capacidades de estos para proponer cambios posibles.

Sin embargo, en la última década ha emergido un creciente cuerpo de investigación en que los profesores dan a sus estudiantes 
la oportunidad de expresar sus opiniones para negociar el plan de estudios (Vergara et al., 2015; Guadalupe y Curther-Smith, 2019a; Oliver y Kirk, 2015; Oliver y Oesterreich, 2013; Manni, Sporre y Ottander, 2013; Traver, Moliner y Sales, 2019; Pérez y Ochoa, 2017). Los resultados han demostrado que esta posibilidad de dar voz a los alumnos y permitir su empoderamiento puede mejorar su compromiso y favorecer un número importante de objetivos afectivos en términos de la mejora de la confianza en los estudiantes, de su cooperación y del incremento de relaciones interpersonales positivas entre personas con diferentes capacidades (Guadalupe y Curther-Smith, 2019a).

La relevancia socio-educacional de este estudio radica en tener en consideración las voces de los estudiantes. Este hecho los posiciona como agentes y no como simples receptores, les otorga sentimientos de pertenencia e inclusión, les ayuda a reflexionar y evaluar sus propios aprendizajes, les ofrece oportunidades para dialogar y proponer ideas y sugerencias que, consideradas por docentes e investigadores, podrían favorecer el mejoramiento de las prácticas pedagógicas.

Enmarcado en este contexto, el objetivo de este estudio consiste en analizar la construcción discursiva de significados en torno a las posibilidades del currículo desde las voces de los estudiantes. Para responder a este objetivo, las preguntas que orientan este trabajo son: (a) ¿Qué recursos utilizan los estudiantes en su construcción discursiva de significados en torno al currículo?; (b) ¿Cuáles son los patrones valorativos desplegados por los estudiantes en su construcción discursiva de significados?; (c) ¿Qué participantes, eventos, procesos y circunstancias son legitimados o deslegitimados por los estudiantes en su construcción discursiva de significados en torno al currículo?; d) ¿Cómo son representados los alumnos y profesores?; (e) ¿Qué transformaciones son posibles a partir de las propuestas de los propios estudiantes?

El estudio del que se da cuenta en este artículo forma parte de una investigación mayor (Prácticas Educativas y Procesos Inclusivos), a través de la cual se busca visibilizar y explicitar los recursos y estrategias mediante las cuales se articulan los diferentes significados en torno al currículum escolar. 
Enmarcado en la mirada semiótico-social de la realidad, el análisis se realiza desde una perspectiva teórico-metodológica que integra los aportes del Modelo de la Valoración y las categorías de estrategias y representación de actores sociales tomados de los Estudios Críticos del Discurso.

Este artículo está organizado en las siguientes secciones: La primera sección comienza con la presentación del marco analítico, que se divide en dos partes: (a) una breve contextualización en torno a la importancia de escuchar las voces de los estudiantes; (b) la perspectiva teórica integrada (como se indicó en el párrafo anterior). En la segunda sección, se describe la metodología. En la tercera sección se presenta el análisis discursivo de los significados construidos por los estudiantes participantes de grupos focales en tres colegios públicos de Chile (en Santiago, Viña del Mar y Alto Hospicio). Finalmente, se resumen los resultados y se presentan las conclusiones destacando las estrategias y macroestrategias utilizadas por los estudiantes en los grupos focales.

\section{Marco teórico-analítico}

\subsection{Importancia de las voces de los estudiantes en la negociación del currículo}

Las tensiones de las escuelas públicas chilenas oscilan entre la mirada mercantilista de las políticas educativas (López et al., 2018) y las demandas de la sociedad para no perder el sentido elemental de "hacer escuela". Este un llamado a repensar, desde los sujetos que ahí habitan, qué es lo que construyen día a día en el acto de educar o de educarse (Grinberg, 2016).

La valoración de la mirada y la voz de los y las estudiantes sobre los fenómenos escolares -y sobre otros fenómenos- toma relevancia en el giro que hacen los nuevos estudios sociales de la infancia (Vergara et al., 2015) cuando se entiende al adultocentrismo como un sistema de dominación que posiciona a niñas, niños y jóvenes en un lugar de subordinación (Figueroa-Grenett, 2017).

En las escuelas las relaciones entre estudiantes y profesores/ as se ven intervenidas por políticas públicas que no representan los 
intereses reales de aprendizaje y, como consecuencia, las acciones que llevan a cabo las autoridades educativas no condicen con las realidades de las comunidades a las cuales educan las escuelas (Conzuelo, 2009; Manni et al., 2013). La pedagogía ha sido predominantemente centrada en el profesor (Cothran, 2013). Los docentes tradicionalmente han tendido a desestimar las habilidades de los estudiantes para pensar en sus propias necesidades (Oliver y Kirk, 2015).

Frente a este panorama se hace relevante preguntar qué quieren aprender las y los estudiantes o cómo valoran su proceso educativo desde sus experiencias y visiones. En la última década ha emergido un creciente cuerpo de investigación en que los profesores dan a sus estudiantes la oportunidad de expresar sus opiniones para negociar el plan de estudios; los resultados han demostrado que esta posibilidad de dar voz a los alumnos y permitir su empoderamiento puede mejorar su compromiso y favorecer un número importante de objetivos afectivos en términos de la mejora de la confianza en los estudiantes, de su cooperación y del incremento de relaciones interpersonales positivas entre personas con diferentes capacidades (Guadalupe y Curther-Smith, 2019a). A la vez, la negociación entre profesores y estudiantes puede favorecer el grado de alineamiento entre el currículo formal y el oculto (Høgdal, Rasche, Schoeneborn, y Scotti, 2019), al tener en cuenta los intereses y necesidades de los alumnos, quienes cooperan con el profesor en la toma de decisiones, mediante la explicitación de los intereses de ambos.

Negociar el currículum no significa que los profesores permitan a los estudiantes tomar todas las decisiones (Oliver y Kirk, 2015; Oliver y Oesterreich, 2013). La negociación implica que los profesores vean a los estudiantes como centro y utilicen métodos basados en el cuestionamiento, facilitando las discusiones, escuchando y respondiendo a los alumnos, valorando sus voces y tomando en cuenta su retroalimentación al momento de tomar decisiones curriculares (Guadalupe y Curtner-Smith, 2019a; Oliver y Kirk, 2015; Enright y O’Sullivan, 2013).

Sin embargo, la negociación del currículo puede ser un proceso difícil para profesores o estudiantes. El temor a la pérdida del control 
puede conducir a un período temporal de desestabilización o de lucha con la idea dudosa del profesor de ceder algo de su poder a los estudiantes. Además a ellos les puede tomar tiempo aprender cómo negociar el poder con sus profesores y entre ellos mismos (Guadalupe y Curtner-Smith, 2019b).

A pesar de esta posibles dificultades la valoración de las voces de los estudiantes puede favorecer el aprendizaje y la enseñanza, así como el empoderamiento de los niños como ciudadanos y agentes de cambio (Manni et al., 2013). La participación activa de los alumnos permite su visibilización y, a la vez, implica una vinculación activa, una interconexión de saberes y una reflexión para la transformación social (Traver, Moliner y Sales, 2019). Por ende, favorece la ciudadanía responsable, con conciencia ética y solidaria en contextos de transformación social (Pérez y Ochoa, 2017).

En síntesis, la problematización de una propuesta curricular y los procesos de negociación del currículo implican una reflexión sobre qué aprender y cómo hacerlo. Esta toma de decisiones requiere apropiación curricular de parte de profesores y estudiantes, quienes se valen de variadas estrategias para consensuar el currículo.

Urbina-García (2019) obtiene variadas evidencias de que en la mayor parte de las investigaciones con niños y niñas se utilizan métodos de entrevistas y observaciones realizadas por adultos/as, por lo que recomiendan, espacios de investigación que amplíen las formas de recoger datos, avanzando hacia el uso de objetivos como fotografías o juegos, entre otros.

En la actual investigación que se informa en este artículo se han explorado formas distintas de recoger datos con estudiantes que permitan dar voz a las experiencias educativas desde sus propias vivencias cotidianas para avanzar hacia propuestas de nuevas posibilidades de aprender, tal como lo muestran los resultados de los grupos focales que se analizan en este estudio. 


\subsection{Perspectiva teórica integrada: Modelo de la Valoración y Estudios Críticos del Discurso}

La construcción discursiva de significados por parte de estudiantes participantes de los grupos focales da cuenta del alineamiento o desalineamiento con determinadas prácticas en tres contextos escolares chilenos diferentes. El análisis de dichos significados es informado desde una perspectiva que integra, por un lado, el Modelo de la Valoración (Martin y White, 2005; Oteíza, 2017; Oteíza, 2016; Oteíza y Pinuer, 2012) y, por otro lado, los Estudios Críticos del Discurso, de los que se toman en consideración específicamente:

a) Tres categorías del inventario sociosemántico de la representación de actores sociales (van Leeuwen, 2008): activación, funcionalización y autorización, que son definidas operacionalmente al final de esta sección.

b) Las categorías de estrategias y macroestrategias (Benke y Wodak, 2003; Wodak, 1999 y 2011; De Cillia et al., 2015).

Los paradigmas epistémicos mencionados se enmarcan en la mirada semiótico-social de la realidad tal como la concibe la Lingüística Sistémica Funcional. Esta define el lenguaje como sistema semiótico social que los hablantes emplean para construir significados en contexto, a través de opciones a diferentes niveles (Halliday, 1994, 2014).

A partir del paradigma funcionalista, surge el Modelo de la Valoración, perspectiva teórico-metodológica que provee una descripción y explicación de los caminos en que el lenguaje es usado para evaluar la experiencia social (Martin y White, 2005) y para dar cuenta de la representación de actores sociales y eventos y procesos históricos (Oteíza y Pinuer, 2016).

Según Martin y White (2005), el Modelo de la Valoración comprende los recursos evaluativos que pueden dividirse en tres grandes regiones semánticas: la ACTITUD, el COMPROMISO, también denominado Involucramiento, y la GRADACIÓN. El sistema de ACTITUD está vinculado tanto con respuestas emocionales como con sistemas de valores culturales. Dentro de esta región semántica, existen tres subsistemas: JUICIO, AFECTO y APRECIACIÓN. El AFECTO se 
vincula con la expresión de sentimientos positivos o negativos con respecto a fenómenos, eventos, procesos, participantes. Estos pueden ser de: Felicidad, Seguridad, Satisfacción o sus contrarios: Infelicidad, Inseguridad, Insatisfacción. El JUICIO se relaciona con la evaluación de personas con respecto a normas sociales institucionalizadas. Los juicios pueden ser de "Estima social" o de "Sanción social". La APRECIACIÓN se vincula con la evaluación en función de principios estéticos y de sistemas axiológicos propios de una sociedad. No obstante, en el presente estudio no es considerado el subsistema de APRECIACIÓN en los términos postulados por Martin y White (2005). En su lugar, se hace uso de la red semántica de APRECIACIÓN para la evaluación de eventos, situaciones y procesos (sociales, políticos y/o económicos), de acuerdo con la reformulación planteada por Oteíza y Pinuer (2012).

El COMPROMISO se vincula con las fuentes, las diferentes voces y con variadas posiciones ideológicas. A mayor incorporación de las mismas en el discurso, se crea una posición heteroglósica y, por el contrario, la ausencia de otras voces genera una monoglosia, en términos bajtinianos, citados por White (2003). El sistema de GRADACIÓN se vincula con el hecho de que las actitudes pueden ser aumentadas o disminuidas en el discurso. De este modo, los significados pueden ser intensificados (Fuerza), o es posible 'agudizar' o 'suavizar' los límites categoriales de un fenómeno experiencial (Foco) usando expresiones como "un tipo de" o "como que". En la presente investigación se considera la red de opciones planteadas por Susan Hood (2010) para el sistema de GRADACIÓN.

Por otra parte, de los Estudios Críticos del Discurso se toma en consideración el inventario sociosemántico de los caminos por medio de los cuales los actores sociales pueden ser representados (van Leeuwen, 2008). A continuación se definen las categorías del inventario presentes en el corpus analizado:

a) La activación ocurre cuando los actores se proponen como seres capaces de acción, sentimiento, volición y racionalización del mundo; son agentes capaces de proponer y ejecutar acciones futuras, las que son socialmente valoradas en forma positiva.

b) La funcionalización ocurre cuando los actores sociales son referidos en términos de una actividad que ellos hacen. 
c) La autorización es la legitimación por referencia a la autoridad en sus diferentes formas, de las cuales se define la que aparece en el corpus, es decir, la autoridad de experto: según los conocimientos o habilidades en un área específica.

También de los Estudios Críticos del Discurso se toma en consideración el concepto de estrategia que denota un plan más o menos preciso adoptado para lograr un determinado objetivo político, psicológico, discursivo o de otro tipo. El estratega intenta anticipar todos aquellos factores que pueden tener un impacto en sus acciones (Wodak, 1999: 78).

La investigadora austríaca reconoce que su interpretación de la estrategia está muy en deuda con la noción de Pierre Bordieu: "la estrategia se relaciona con la existencia de un conjunto de prácticas destinadas a alcanzar un objetivo y que han perdurado y se han utilizado sistemáticamente a lo largo de los años sin ser asumidas conscientemente" (Bordieu: 1994: 49).

Wodak (1999: 79) considera que a nivel macro se pueden distinguir diferentes tipos de macroestrategias en la construcción discursiva de la identidad nacional (tema central de su investigación) aplicables a otros corpus. Ella las denomina macroestrategias discursivas porque estas hacen uso de distintas estrategias. Estas macroestrategias corresponden a las principales macrofunciones sociales: construcción, transformación, perpetuación y demonio o desmantelamiento. Aunque analíticamente distinguibles entre sí, estas macroestrategias ocurren más o menos simultáneamente y se entrelazan en actos discursivos concretos.

Cabe entonces explicar el vínculo entre las mencionadas estrategias y macroestrategias, por un lado, y el Modelo de la Valoración, por otro lado, en relación con los posicionamientos de los estudiantes en torno al currículo. La valoración de este en las distintas prácticas sociales discursivas es realizada desde determinados puntos de vista, desde ciertas evaluaciones de JUICIO, AFECTO y APRECIACIÓN con diferentes niveles de COMPROMISO.

Los recursos verbales se acumulan conformando lo que se denominan prosodias valorativas (Martin y White, 2005). Estas pueden 
funcionar como estrategias de legitimación o deslegitimación de participantes o actores sociales, procesos y eventos. A la vez, dichas estrategias pueden conformar macroestrategias de transformación o perpetuación del status quo (Benke y Wodak, 2003; Wodak, 1999 y 2011; De Cillia et al., 2015). Las macroestrategias de perpetuación tratan de mantener y reproducir grupos, imágenes u otros artefactos discursivos ya establecidos. En cambio, las macroestrategias de transformación intentan cambiar un status quo en algo diferente. Estas categorías, presentes en el discurso de los estudiantes en torno al currículo escolar chileno, son profundizadas en el análisis y en las conclusiones de este estudio.

\section{Metodología}

De acuerdo con los objetivos de esta investigación, el método de análisis es cualitativo (Angouri, 2010; Denzin y Lincoln, 2012). El diseño es flexible (Mendizábal, 2006). El estudio del que se da cuenta está enmarcado en una investigación mayor. Sin embargo, el corpus de este artículo está conformado por tres grupos focales ${ }^{4}$ en tres colegios públicos de Chile (en Santiago, Viña del Mar y Alto Hospicio). Para su realización se contaron con los respectivos consentimientos y asentimientos en el caso de los menores de 14 años.

La transcripción de los grupos focales es realizada por las investigadoras con el propósito de familiarizarse más profundamente con los recursos utilizados por los informantes en la construcción discursiva de sus propios posicionamientos.

4. La recolección de datos fue realizada a través de un grupo focal, cuyos participantes fueron en promedio 8 estudiantes de diferentes cursos de Quinto Básico a Cuarto Medio. Ellos participaron de una actividad denominada: "El superpoder de soñar la escuela". Entre todos y todas debían generar un horario de clases de la escuela ideal para ellos, con actividades de su interés y explicitando los actores sociales vinculados con el establecimiento educacional que podrían participar en cada taller, curso o actividad propuesta. Los estudiantes debían plasmar el horario en un diseño en grande donde figuraban todos los días de la semana y horas del día. Los alumnos también disponían de imágenes o dibujos que pudiesen representar las actividades, la metodología de funcionamiento, las personas involucradas dentro de cada establecimiento educacional. Para el registro de la recogida de datos se utilizaron grabaciones en audio, videograbaciones y registro fotográfico. 
La unidad de análisis es el complejo de cláusulas ${ }^{5}$, compuesto por fragmentos de los grupos focales que incluyen los datos más significativos en cuanto a información detallada y en términos de evaluaciones.

El análisis está constituido por dos fases. La primera es descriptiva y de naturaleza cualitativa, no sólo a nivel léxico-gramatical sino también discursivo-semántico, e intenta identificar qué y quiénes son evaluados (actores sociales, procesos); cómo se evalúan (sistemas semánticos ACTITUD y GRADUACIÓN); y las fuentes de evaluación (sistema de COMPROMISO).

En la segunda fase analítica, de naturaleza interpretativa, por una parte, como herramientas heurísticas se consideran las prosodias valorativas (Martin y White, 2005; Hood, 2010; Oteíza y Pinuer, 2016) y el funcionamiento de estas como estrategias de legitimación o deslegitimación. En esta fase se trasciende el plano lingüístico y se incorporan las diferentes manifestaciones ideológicas del fenómeno social de las prácticas escolares analizadas, usando las siguientes herramientas analíticas: activación, funcionalización y autorización; estrategias discursivas de legitimación y deslegitimación de actores, procesos y eventos; macroestrategias de transformación.

Por otra parte, en la fase interpretativa se analiza el cambio de significados en torno al currículo escolar en las tres escuelas con el fin de visibilizar variadas formas de transformar o perpetuar las prácticas en dichos contextos escolares.

\section{Análisis y discusión}

En esta sección se analiza cómo los estudiantes construyen discursivamente su posicionamiento ideológico de acuerdo con sus propias vivencias en cada contexto en que emergen sus discursos.

5. "El complejo de cláusulas se forma a partir de una secuencia lineal de nexos de cláusulas; cada nexo consta de un par de cláusulas relacionadas. Muchos complejos de cláusulas son secuencias lineales de este tipo pero también a menudo se agrupan o anidan" (Halliday y Matthiessen, 2004: 376). 
En el análisis se pueden observar dos tipos de voces: voz de recopilador y voz de evlauador (Martin y White, 2005: 178; Coffin, 2006: 154). En el primer caso, los estudiantes sólo describen o narran pero sin evaluar o lo hacen implícitamente. Por el contrario, en el segundo caso, los estudiantes construyen discursivamente sus evaluaciones en torno a variados tópicos vinculados con el currículo escolar. Estas voces son también consideradas estrategias retóricas, las que muestran diferentes posicionamientos ideológicos y crean diversos niveles de COMPROMISO adoptados por los estudiantes, como se percibe en los ejemplos 1,2 y 3 .

Ejemplo $1^{6}$

Nos gustaría tener clases especiales y más lúdicas de Matemática y Lenguaje. Queremos aprender de profesores que les guste lo que enseñan. Así como ellos nos enseñan, que ellos aprendan de nosotros. (Alto Hospicio)

Ejemplo 2

Nos gustaría leer textos interesantes que nos gusten más. Necesitamos más libros gruesos de acción y romances como: Ángeles caídos, Bajo la misma estrella, La Reina Roja. (Santiago)

Ejemplo 3

Además sería buenísimo tener un laboratorio y pasar mucho rato haciendo experimentos. La profesora tendría que ser experta. (Santiago)

Por un lado, los estudiantes evalúan las clases de Matemática y Lenguaje a través de APRECIACIONES inscritas, positivas (“especiales", "lúdicas", "interesantes"), graduadas por Cuantificación en el segundo caso ("más"). La forma condicional "gustaría" permite instanciar el ideal que los alumnos tienen respecto de las clases, cuya metodología

6. Las marcas de notación en los ejemplos son las siguientes:

Procesos en negrita (sólo cuando el proceso incluye evaluaciones // Evaluación inscrita: negrita y subrayada // Evaluación evocada: negrita y cursiva // Polaridad negativa y Contra-argumentación

ACTITUD

AFECTO (ej. amor: AFECTO positivo inscrito de Felicidad)

JUICIO (ej. golpiza brutal: JUICIO negativo inscrito de Sanción Social, carencia de Integridad)

APRECIACIÓN (ej. desorden: APRECIACIÓN inscrita, Alta Conflictividad, Impacto Negativo, Deslegitimación)

GRADACIÓN: FOCUS (mayúsculas) // fuerza (minúsculas subrayadas) 
produzca placer y entretenimiento en los estudiantes. A través de procesos mentales afectivos ("queremos", "guste", "gusten") y mentales cognitivos ("enseñan", "aprendan"), los alumnos expresan su posicionamiento a favor de docentes comprometidos con un proceso de mutuo aprendizaje, en el que los estudiantes cumplan un doble rol: aprender de sus docentes y, a la vez, enseñar a ellos también; una forma de hacerlo es dando a los profesores datos de libros que a ellos les interesen, como se percibe en el ejemplo 2. En el caso específico de la clase de Lenguaje, los estudiantes evalúan ciertos libros de su interés por medio de APRECIACIONES inscritas, positivas ("de acción y romances"). Por otro lado, los alumnos evalúan las clases de Química también como un ideal a través de la forma condicional "sería" y de un coupling de una APRECIACIÓN inscrita, positiva ("buenísimo"), graduada por Fuerza, Cuantificación ("mucho"), que está combinada con un JUICIO inscrito, positivo, de Estima Social de Capacidad ("experta"), que instancia el posicionamiento de los estudiantes a favor de profesores avezados y experimentados que los acompañen en el proceso de aprender en forma práctica en un laboratorio.

Teniendo en consideración las categorías de actores, en los términos planteados por van Leeuwen (2008), en los tres ejemplos analizados es posible observar la activación, ya que los alumnos muestran su capacidad propositiva y, a la vez, su deseo de convertirse en agentes de su propio aprendizaje ayudados con la mediación de profesores lúdicos y expertos, a la vez. Además los alumnos se autoperciben en dos funciones diferentes: aprender y enseñar. De este modo, su perspectiva responde a la categoría de van Leeuwen de funcionalización, ya que tanto profesores como alumnos serían actores sociales que comparten el proceso de enseñanza y aprendizaje.

El mencionado requisito de experticia es evaluado en los diferentes colegios en relación a otros tipos de conocimientos, como se advierte en los ejemplos 4 y 5 .

Ejemplo 4

Nos gustaría mucho dibujar y pintar, por ejemplo, en un muro del colegio. Eso nos serviría mucho para aprender a expresarnos y desarrollar la creatividad. (Alto Hospicio) 


\section{Ejemplo 5}

Nos gustaría mucho hacer algo bonito. Necesitaríamos un buen profesor experto y motivado como Leonardo da Vinci. (Santiago)

Por una parte, los estudiantes instancian su ideal a través de prosodias saturadas en las que se repiten las formas condicionales ("gustaría", "serviría", "necesitaríamos") y la GRADACIÓN por Fuerza, Cuantificación ("mucho"). Por otra parte, los alumnos evalúan a través de un coupling de APRECIACIONES inscritas positivas ("creatividad", "bonito") y de JUICIOS inscritos de Estima Social, de Capacidad ("experto", "motivado"), que les permiten expresar la necesidad de docentes legitimados por su experticia, la que es instanciada por un símil ("como Leonardo da Vinci").

Los estudiantes se perciben a sí mismos como agentes capaces de desarrollar sus propias potencialidades creativas, apoyados por la mediación de sus profesores. En términos de van Leeuwen (2008) los alumnos son representados por medio de la activación y los profesores, a través de la autorización, es decir, la legitimación por referencia a la autoridad de experto en este caso.

Además de requerir expertos para asignaturas científicas, humanísticas o artísticas visuales, también los estudiantes evalúan la experticia en el área musical, como se observa en el siguiente ejemplo.

Ejemplo 6

Sería entretenido tener clases especiales de música. Sería divertido empezar el día con música en un taller con cuatro profesores diferentes para aprender distintos instrumentos. Así aprenderíamos a tocar guitarra eléctrica, piano, violín, batería. (Santiago)

Los estudiantes evalúan las clases ideales de música por medio de formas condicionales ("sería", "aprenderíamos") y de APRECIACIONES inscritas, positivas ("entretenido", "divertido", "distintos"). A través de su lenguaje evaluativo los alumnos valoran positivamente la diversidad de expertos en diversos instrumentos musicales a través de un JUICIO inscrito, positivo de Estima Social de Capacidad (“diferentes"). 
Teniendo en cuenta la representación de los actores sociales, en términos de van Leeuwen (2008), nuevamente en este ejemplo los alumnos son representados por medio de la activación, como agentes propositivos e interesados en su propio desarrollo artístico musical. A su vez, desde la perspectiva estudiantil, la propuesta representa a sus profesores por medio de la funcionalización, debido al rol de maestros expertos en diferentes instrumentos. Al mismo tiempo, los docentes son representados por medio de la categoría de autorización debido a su conocimiento y habilidad como expertos en la ejecución de distintos instrumentos musicales.

Ese requerimiento de experticia también se percibe en el siguiente ejemplo 7, vinculado con los juegos y con el ámbito deportivo.

Ejemplo 7

Podríamos tener clase de ping-pong para aprender con un joven experto porque nadie sabe mucho. También sería bueno tener una clase de skate con un profesional, con un campeón de skate. (Santiago)

Los estudiantes instancian su ideal deportivo a través de formas condicionales ("podríamos", "sería"), de APRECIACIONES inscritas, positivas ("bueno") y de JUICIOS inscritos, positivos, de Estima Social, Capacidad (“experto", "profesional”, “campeón”). La polaridad negativa ("nadie") refuerza la necesidad de un experto también en lo deportivo.

Además los estudiantes realizan una serie de propuestas vinculadas especialmente con los deportes y juegos, como se percibe en ejemplos $8,9,10$ y 11 .

Ejemplo 8

El deporte sería bueno para bajar de peso. (Alto Hospicio)

Ejemplo 9

Sería bueno tener un tiempo en la tarde para recorrer el cerro en bicicleta. (Santiago)

Ejemplo 10

Es bueno porque así aprendemos bien a cuidar el medio ambiente. Nos gustaría mucho hacer ejercicios, como andar en patineta. En esta actividad 
participaríamos todos. Los niños y los funcionarios podríamos jugar taca-taca y ajedrez. Nosotros podríamos elegir libremente y jugar a lo que nosotros queramos. (Alto Hospicio)

\section{Ejemplo 11}

Sería mejor que los juegos (skate, taca-taca, ping-pong) estuvieran en el patio, unos al lado de otros, dentro del colegio. También jugaríamos con un ajedrez gigante que estaría en el colegio en el patio o en uno de los pasillos. Jugar ajedrez es difícil. (Santiago)

Los estudiantes evalúan sus propuestas deportivas, planteadas como actividades ideales, mediante formas condicionales ("sería". “jugaríamos", "gustaría”" “participaríamos”, "podríamos”, "jugaríamos”, “estaría”) y APRECIACIONES inscritas, positivas ("bueno", "bien”, "mejor"), graduadas en algunos casos por Fuerza, Intensificación ("libremente" o Cuantificación ("mucho"). Los alumnos no sólo evalúan las ventajas de los deportes o juegos propuestos sino también el lugar de los mismos ("mejor"), el tamaño ("gigante") y el grado de dificultad ("difícil). Como se percibe en los ejemplos, el lenguaje evaluativo predominante está conformado mayoritariamente por APRECIACIONES inscritas, positivas ("mejor") o negativas ("difícil"), según los casos. Sin embargo, algunos juegos o deportes son evaluados a través de JUICIOS de Estima Social de Capacidad (“experto", “campeón”, "profesional”).

Nuevamente en estos ejemplos los alumnos son representados por medio de la activación, como agentes propositivos y deseosos de participar en juegos, deportes y variadas actividades en contacto con la naturaleza que favorecerían su desarrollo físico y promoverían una vida más saludable, beneficiada también por una propuesta respecto de la comida. Nuevamente, los docentes son representados por medio de la categoría de autorización debido a su experticia deportiva, en juegos específicos y también en alimentación, como se percibe en los ejemplos siguientes.

Este mismo requerimiento se observa en los ejemplos 12 y 13, vinculados con la participación activa de los estudiantes en la preparación de la comida para toda la comunidad. 


\section{Ejemplo 12}

Sería bueno que aprendiéramos a cocinar nosotros y lo hiciéramos todos los días. Podría ser mejor un almuerzo en forma de banquete o tenedor libre donde se ofrezca un menú a elección. (Alto Hospicio)

\section{Ejemplo 13}

Nosotros cocinaríamos para todo el colegio, tanto cosas saludables como alimentos no tan saludables. Necesitaríamos un adulto experto que nos guíe. Cocinaríamos papas fritas, lasagnas. (Santiago)

En los colegios técnicos donde se hicieron los grupos focales, los estudiantes de Gastronomía evaluaron su propia propuesta a través de una APRECIACIÓN inscrita, positiva ("bueno", "mejor", "libre"). Además los alumnos evaluaron a sus profesores de la especialidad a través de un JUICIO inscrito, positivo, de Estima Social de Capacidad ("experto") que les enseñaran diferentes tipos de comidas. A la vez, los estudiantes instancian su ideal mediante formas condicionales ("sería", "cocinaríamos", "necesitaríamos") y mediante el Cuantificador "todo" para reforzar los destinatarios de sus productos alimenticios.

La presencia de un experto aparece también en otras propuestas de los estudiantes, como Infórmate, tal como se observa en el ejemplo 14.

Ejemplo 14

Este sería un espacio para todos, considerando la edad de cada uno, para informarse sobre lo que pasa en mi país y en el mundo. Ej. Problemas de género, aborto. Es importante considerar la edad porque no sería conveniente hablarle a un niño de segundo básico sobre el aborto. No debería ser sólo una conversación entre nosotros sino que nos acompañara una persona experta. (Alto Hospicio)

Los estudiantes evalúan su propia propuesta a través de una APRECIACIÓN inscrita, positiva ("importante") y el cuantificador "todos" para recalcar la posibilidad de participación de toda la comunidad educativa de acuerdo con la edad de cada actor social. A la vez, los alumnos evalúan al responsable de la actividad mediante un JUICIO inscrito, positivo de Estima Social, de Capacidad ("experta"). 
En términos de van Leeuwen (2008), los alumnos son representados mediante la activación pero no solo como agentes que buscan su propia participación e información sino como difusores e impulsores de toda la comunidad escolar. Como en todos los ejemplos anteriores, el docente mediador es representado mediante la autorización, por su experticia.

Además los estudiantes buscan la concreción de un sentido comunitario, al realizar propuestas como se ve en el ejemplo 15.

Ejemplo 15

Sería interesante incluir un espacio diario donde se aprenda a respetar al otro, aprender de él y valorar la diversidad. (Alto Hospicio)

Los estudiantes evalúan su propuesta ideal por medio de una forma condicional ("sería") y de APRECIACIONES inscritas positivas ("interesante", "diversidad").

Los alumnos son representados mediante la activación como agentes que buscan la visibilización de las opiniones minoritarias.

Además de los talleres ya mencionados (muralismo, cocina, inclusión), los alumnos proponen otros, que impliquen un mayor contacto con la comunidad, tal como se observa en los siguientes 16, 17 y 18 .

Ejemplo 16

El Taller de Astronomía sería útil para aprender del cielo. (Alto Hospicio)

Ejemplo 17

Es bueno aprender Astronomía viendo videos, leyendo, siguiendo instrucciones, visitando lugares como el Planetario. (Santiago)

Ejemplo 18

Podríamos venir de noche a la escuela a observar las estrellas. (Viña del Mar)

Los estudiantes evalúan su propuesta a través deAPRECIACIONES inscritas, positivas ("útil”, "bueno") y mediante una GRADACIÓN, Extensión Temporal ("de noche") que muestra el compromiso de asistencia en horario extraescolar para cumplir con el objetivo del taller sugerido. 
También los estudiantes utilizan lenguaje valorativo en relación con posibles salidas culturales, como se observa en los ejemplos 19, 20,21 y 22 .

\section{Ejemplo 19}

No queremos aprender sólo dentro de la escuela sino que también fueran actividades al aire libre y fuera de nuestra comunidad escolar. Podríamos visitar museos, sería conveniente para aprender mejor. También sería en-

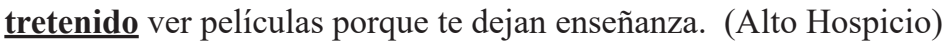

\section{Ejemplo 20}

Ir al cine es bueno para compartir con la familia o con los amigos. Si fuera con mi familia vería una película bonita. Veríamos películas de actualidad. (Santiago)

\section{Ejemplo 21}

Podríamos ir al planetario. Ese es un lugar diferente de la escuela y donde podemos aprender también. Iría al Platenario con mi familia para enseñarles si tuviera plata pero estoy en bancarrota. (Santiago)

\section{Ejemplo 22}

O podríamos ir a limpiar la playa en una acción más comunitaria. (Santiago)

Los estudiantes evalúan las actividades fuera del edificio escolar, tales como visitas a museos, al cine o al planetario, a través de formas condicionales ("sería", "vería", "veríamos", "podríamos") y de APRECIACIONES inscritas de Valuación positiva ("diferente", "conveniente", "entretenida", "bueno") o de Reacción positiva ("bonita", "de actualidad"). A la vez, los alumnos valoran las salidas con fines comunitarios, como la limpieza de la playa, mediante una APRECIACIÓN inscrita, de Valuación positiva ("comunitaria") graduada por Fuerza, Cuantificación ("más”). Un caso excepcional en el uso de lenguaje evaluativo vinculado con salidas fuera del establecimiento escolar es la del estudiante que desea incluir y hacer partícipe a su familia pero instancia su ideal frustrado a través de una APRECIACIÓN inscrita, negativa vinculada con la situación económica ("en bancarrota") que constituye un impedimento para el cumplimiento de dicho deseo. 
Los estudiantes proponen actividades motivadoras y que llenen de alegría el diario vivir en las escuelas, tal como se percibe en el ejemplo 23.

\section{Ejemplo 23}

Podríamos tener un taller de rapeo no obligatorio después de clases. Así vendrían todos los días con ánimo. Terminaríamos el día con rapeos para improvisar. Participarían los que quieran, niños y adultos que quieran. No a todos les gusta lo mismo. Por ejemplo, los Inspectores son medio fomes, son COMO del siglo pasado, son pesados. (Santiago)

Los alumnos evalúan su propuesta posible través de formas condicionales ("podríamos", "participarían"), de APRECIACIONES inscritas, positivas ("no obligatorio") y de GRADACIÓN, Cuantificación ("todos") para instanciar el carácter de libre opción y de motivación del taller, conscientes de la diversidad de gustos. A la vez, los estudiantes evalúan a los Inspectores por medio de AFECTOS inscritos, negativos de Insatisfacción ("fomes", "pesados"), graduados por Fuerza ("medio") y por FOCO ("COMO”) para instanciar la posible no participación de los Inspectores en este taller de rapeo e improvisación por pertenecer a otra época y por no tener una relación cercana con ellos.

En todas las propuestas de los estudiantes predomina la voz de evaluador, como se percibe en la instanciación del interés por diferentes talleres con el mismo objetivo de motivar a la comunidad escolar y proporcionale alegría en el diario vivir, tal como se observa en el ejemplo 24 .

\section{Ejemplo 24}

También podríamos hacer karaoke un Taller de danza de una hora, donde un adulto enseña bien a los niños. Sería más entretenido tener música enfocada en el baile como zumba pero más divertido, más animado tanto para adultos como para estudiantes. (Santiago)

Los estudiantes evalúan sus sugerencias de canto y danza por medio de formas condicionales ("podríamos") y de APRECIACIONES inscritas, positivas ("entretenido", "divertido", "animado"), graduadas por Fuerza, Cuantificación ("más"). De este modo los alumnos legitiman propuestas musicales que sean motivadoras para los distintos grupos etarios de la comunidad educativa en su totalidad. 
Según la categorización de van Leeuwen (2008), los alumnos son representados mediante la activación como agentes que buscan la ampliación del abanico de opciones para favorecer el potencial de cada uno según los propios intereses: astronomía, cine, playa, cine, rapeo, danza y teniendo en consideración las posibles actividades fuera del aula y de la escuela, en contacto con la naturaleza y con la comunidad citadina con todas las posibilidades que esta ofrece.

Para cerrar esta sección de análisis y resultados, la tabla 1 sintetiza los ítems evaluativos en las distintas regiones semánticas de los diferentes tópicos, sobre los que se profundiza en la discusión y conclusiones.

Tabla 1 - Prosodias valorativas

\begin{tabular}{|c|c|c|c|c|c|}
\hline TÓPICOS & $\begin{array}{l}\text { ÍTEMS } \\
\text { VALUA- } \\
\text { TIVOS }\end{array}$ & AFECTO & JUICIO & $\begin{array}{l}\text { APRECIA- } \\
\text { CIÓN }\end{array}$ & GRADACIÓN \\
\hline $\begin{array}{l}\text { Matemática, } \\
\text { Lenguaje, } \\
\text { Química }\end{array}$ & $\begin{array}{l}\text { Especiales } \\
\text { Lúdicas } \\
\text { Interesantes } \\
\text { Buenísimo } \\
\text { Experta }\end{array}$ & & E.S. + Capacidad & $\begin{array}{l}\text { +Valuación } \\
\text { +Valuación } \\
\text { +Valuación } \\
\text { +Valuación }\end{array}$ & $\begin{array}{l}\text { Más } \\
\text { (Fuerza, } \\
\text { Cuantificación) }\end{array}$ \\
\hline Artes visuales & $\begin{array}{l}\text { Creatividad } \\
\text { Bonito } \\
\text { Experto }\end{array}$ & & E.S. + Capacidad & $\begin{array}{l}\text { +Valuación } \\
\text { +Valuación }\end{array}$ & $\begin{array}{l}\text { Mucho } \\
\text { (Fuerza, } \\
\text { Cuantificación) }\end{array}$ \\
\hline Artes musicales & $\begin{array}{l}\text { Entretenido } \\
\text { Especiales } \\
\text { Divertido } \\
\text { Distinto } \\
\text { Diferentes } \\
\text { Experto }\end{array}$ & & E.S. + Capacidad & $\begin{array}{l}\text { +Valuación } \\
\text { +Valuación } \\
\text { +Valuación } \\
\text { +Valuación } \\
\text { +Valuación }\end{array}$ & \\
\hline Juegos & $\begin{array}{l}\text { Bueno } \\
\text { Difícil } \\
\text { Experto } \\
\text { Profesional } \\
\text { Campeón }\end{array}$ & & $\begin{array}{l}\text { E.S. + Capacidad } \\
\text { E.S. + Capacidad } \\
\text { E.S. + Capacidad } \\
\text { E.S. + Capacidad }\end{array}$ & +Valuación & \\
\hline Deportes & $\begin{array}{l}\text { Bueno } \\
\text { Bien } \\
\end{array}$ & & & $\begin{array}{l}\text { + Valuación } \\
\text { +Valuación }\end{array}$ & \\
\hline Cocina & $\begin{array}{l}\text { Bueno } \\
\text { Mejor } \\
\text { Libre } \\
\text { Saludables } \\
\text { No } \\
\text { saludables } \\
\text { Experto } \\
\end{array}$ & & E.S. + Capacidad & $\begin{array}{l}\text { + Valuación } \\
\text { +Valuación } \\
\text { +Valuación } \\
\text { +Valuación } \\
\text {-Valuación }\end{array}$ & $\begin{array}{l}\text { Todos } \\
\text { (Fuerza, } \\
\text { Cuantificación) }\end{array}$ \\
\hline Infórmate & $\begin{array}{l}\text { Importante } \\
\text { Experta }\end{array}$ & & E.S. + Capacidad & + Valuación & $\begin{array}{l}\text { Todos } \\
\text { (Fuerza, } \\
\text { Cuantificación) }\end{array}$ \\
\hline
\end{tabular}




\begin{tabular}{|c|c|c|c|c|c|}
\hline TÓPICOS & $\begin{array}{c}\text { ÍTEMS } \\
\text { VALUA- } \\
\text { TIVOS }\end{array}$ & AFECTO & JUICIO & $\begin{array}{l}\text { APRECIA- } \\
\text { CIÓN }\end{array}$ & GRADACIÓN \\
\hline $\begin{array}{l}\text { Taller de } \\
\text { inclusión }\end{array}$ & $\begin{array}{l}\text { Interesante } \\
\text { Diversidad }\end{array}$ & & & $\begin{array}{l}\text { + Valuación } \\
\text { +Valuación }\end{array}$ & \\
\hline $\begin{array}{l}\text { Taller de } \\
\text { Astronomía }\end{array}$ & Útil & & & + Valuación & $\begin{array}{l}\text { De noche } \\
\text { (Fuerza, } \\
\text { Cuantificación, } \\
\text { Extensión } \\
\text { Temporal) } \\
\end{array}$ \\
\hline $\begin{array}{l}\text { Taller de } \\
\text { karaoke o } \\
\text { baile, rapeo }\end{array}$ & $\begin{array}{l}\text { Bien } \\
\text { Entretenido } \\
\text { Divertido } \\
\text { Animado } \\
\text { Fomes } \\
\text { Pesados }\end{array}$ & $\begin{array}{l}\text {-Infelicidad } \\
\text {-Insatisfacción }\end{array}$ & & $\begin{array}{l}\text { + Valuación } \\
\text { + Valuación } \\
\text { +Valuación } \\
\text { +Valuación }\end{array}$ & $\begin{array}{l}\text { Más (Fuerza, } \\
\text { Cuantificación) } \\
\text { Más (Fuerza, } \\
\text { Cuantificación) } \\
\text { Más (Fuerza, } \\
\text { Cuantificación) } \\
\text { COMO } \\
\text { (FOCO) }\end{array}$ \\
\hline $\begin{array}{l}\text { Salidas } \\
\text { culturales }\end{array}$ & $\begin{array}{l}\text { Conveniente } \\
\text { Entretenido } \\
\text { Mejor } \\
\text { Bueno } \\
\text { Bonita } \\
\text { Actual } \\
\text { Diferente } \\
\text { Comunitaria }\end{array}$ & & & $\begin{array}{l}\text { + Valuación } \\
\text { +Valuación } \\
\text { +Valuación } \\
\text { +Valuación } \\
\text { +Valuación } \\
\text { +Valuación } \\
\text { +Valuación } \\
\text { +Valuación }\end{array}$ & $\begin{array}{l}\text { Más (Fuerza, } \\
\text { Cuantificación) }\end{array}$ \\
\hline
\end{tabular}

\section{Conclusiones}

En la construcción discursiva de los estudiantes de los tres colegios predominan las APRECIACIONES positivas referidas a sus propuestas y los JUICIOS positivos vinculados con la experticia docente. Paralelamente se advierte la casi ausencia de AFECTOS, excepto en la referencia a los inspectores, evaluados a través de AFECTOS inscritos, negativos, de Infelicidad ("fomes", "pesados").

En términos de la Semiótica Social, los informantes, a través de diversas opciones del lenguaje, instancian sus críticas al sistema escolar, como elemento dado, a través de la voz del reportero. Sin embargo, pese a su corta edad, por medio de la voz del evaluador, proponen elementos nuevos, cambios muy concretos, especialmente a través de las evaluaciones mencionadas que se combinan y acumulan conformando prosodias valorativas. Estas funcionan como estrategias de legitimación o deslegitimación y como macroestrategias de transformación o perpetuación del status quo. 
Por un lado, los estudiantes legitiman, en primer lugar, las clases motivadoras, lúdicas, creativas, a cargo de expertos. A la vez, en segundo lugar, los alumnos legitiman los Talleres nuevos que proponen: cocina, astronomía, rapeo, baile entretenido, karaoke. A estos -se suman dos talleres que buscan la mayor información como así también la diversidad. Tal es el caso de los Talleres: Infórmate e Inclusión. Finalmente, en tercer lugar, los alumnos legitiman las salidas culturas a museos, cine, planetario, playa, entre otras. Por otro lado, los estudiantes deslegitiman las clases con metodologías pasivas y los Inspectores poco afectuosos y motivados.

Finalmente, es posible concluir que los informantes de las tres escuelas hacen uso de macroestrategias de transformación del status $q u o$, al realizar propuestas en dos sentidos. Por un lado, a través de clases con metodologías más variadas, personal experto, más cercano y divertido, buscan transformar la situación educativa de sus colegios. Por otro lado, realizan nuevas propuestas tendientes a una mayor motivación, por medio de actividades en la escuela y fuera de ella, en contacto con la comunidad y como una forma de extender y modificar los contenidos curriculares también.

De esta manera, los estudiantes responden a las preguntas ¿qué enseñar? (temas de actualidad del país y del mundo, como género, aborto y actividades vinculadas con el arte, los deportes y la recreación) ¿cómo enseñar? (con temas acordes con las distintas edades y metodologías activas) y ¿dónde enseñar? (al interior y exterior de la sala de clase y de la escuela) teniendo en cuenta los aportes de sus alumnos.

La relevancia socio-educacional de este estudio radicó en tener en consideración las voces de los estudiantes quienes, frente a la posibilidad de participar en la reflexión del grupo focal, asumieron su propio empoderamiento, cuestionando la realidad pedagógica vivida y, a la vez, adoptando una actitud propositiva. La investigación abre un camino hacia una educación no adultocéntrica en la que se visibiliza a las minorías y, en general, a los estudiantes, cuyas propuestas podrían favorecer el mejoramiento de las prácticas pedagógicas. 


\section{Referencias}

ANGOURI, Jo. 2010. Quantitative, qualitative or both? Combining methods in linguistic research. In: Nige EDLEY; Lia LITOSSELITI (eds.). Contemplating interviews and focus groups. Londres: Continuum.

BAEZA, Patricia. 2017. Construcción de memorias que compiten sobre el pasado chileno reciente por adultos que vivieron ese período en Chile o en el exilio. Discurso \& Sociedad, 11(3):433-457.

BENKE, Gertraud; WODAK, Ruth. 2003. The Discursive Construction of Individual Memorias. How Austrian 'Wehrmacht's soldiers remember WWII. In: Jim MARTIN; Ruth WODAK (eds.). Re/Reading the Past. Critical and Functional Perspectives on Time and Value. Amsterdam, Philadelphia: Benjamins.

BOURDIEU, Pierre. 1994. Las estrategias de la reproducción social. Madrid: Siglo Veintiuno.

COFFIN, Caroline. 2006. Historical discourse: the language of time, cause and evaluation. London, New York: Continuum.

CONZUELO, Sandra. 2009. La voz de los estudiantes. Perfiles Educativos, Vol. XXXI, núm. 125: 117-121.

COTHRAN, Donetta. 2013. "And I hope you see things that startle you": What students can teach us about physical education. Kinesiology Review, 2: 76-80.

DE CILLIA, Rudolf; REISIGL, Martin; WODAK, Ruth. 2015. "La construcción discursiva de identidades nacionales". Andamios. Revista de Investigación Social 12.27: 153-191.

DENZIN, Norman; LINCOLN, Yvonna. 2012. Manual de Investigación Cualitativa. Barcelona: Gedisa.

ENRIGHT, Eimear; SULLLIVAN, Mary. 2013. "Now I'm magazine detective the whole time": Listening and responding to young people's complex experiences of popular physical culture. Journal of Teaching in Physical Education, 32: 394-418.

FAIRCLOUGH, Norman. 2010. Critical Discourse Analysis: The Critical Study of Language. London y New York: Routledge.

FIGUEROA-GRENETT, Claudio. 2018. La acción política de niños, niñas y jóvenes en Chile: cuerpos, performatividad y producción de subjetividad. Revista Latinoamericana de Ciencias Sociales, Niñez y Juventud, 16(1): 199-212. oi:10.11600/1692715x.16111

GRINBERG, Silvia. 2016. Elogio de la transmisión. La escolaridad más allá de las sociedades de aprendizaje. Polifonías Revista de Educación, año V - No 8: 71-94. 
GUADALUPE, Tasha; CURTNER-SMITH, Matthew, 2019a. 'It's nice to have choices': influence of purposefully negotiating the curriculum ion the students in one mixed-gender middle school class and their teacher. Sport, Education and Society, 24.3: 1-13.

.2019b. "We know what we like to do": Effects of purposefully negotiating the curriculum on the girls in one middle school class and their teacher. Journal of Teaching in Physical Education, 39.2.: 147-155.

HALLIDAY, Michael. 1994. An introduction to functional grammar. Londres: Edward Arnold.

. 2014. Halliday's Introduction to Functional Grammar. NY: Routledge.

HALLIDAY, Michael; Matthiessen, Christian. 2004. An Introduction to Functional Grammar. London: Arnold.

HøGDAL, Catharina; Rasche, Andreas; Schoeneborn, Dennis; Scotti, Levinia. 2019. Exploring Student Perceptions of the Hidden Curriculum in Responsible Management Education. Journal of Business Ethics: 1-21.

HOOD, Susan. 2010. Appraising Research: Evaluation in Academic Writing. New York: Palgrave Macmillan.

LÓPEZ, Verónica.; GONZÁLEZ, Pablo; MANGHI, Dominique; ASCORRA, Paula; OYANEDEL, Juan Carlos. 2018. Políticas de Inclusión Educativa en Chile: Tres Nudos Críticos. Archivos analíticos de politicas educativas, 26 (157): 3-12.

MARTIN, Jim. 2003. Introduction. Text, 23(2): 171-181.

MARTIN, Jim; White, Peter. 2005. The Language of Evaluation. Hampshire: Palgrave.

MENDIZÁBAL, Nora. 2006. Los componentes del diseño flexible en la investigación cualitativa. En: Irene VASILACHIS. Coord. Estrategias de investigación cualitativa. Buenos Aires: Gedisa.

OLIVER, Kimberly; KIRK, David. 2015. Girls, gender and physical education: an activist approach. New York: Routledge.

OLIVER, Kimberly; OESTERREICH, Heather. 2013. Student-centered inquiry as curriculum as a model for field-based teacher education. Journal of Curriculum Studies, 45: 394-417.

OTEÍZA, Teresa. 2017. The Appraisal Framework and Discourse Analysis. In: Tom Bartlett; Gerard O'Grady (eds.). The Routledge Handbook of Systemic Functional Linguistics. London: Routledge.

OTEÍZA, Teresa; PINUER, Claudio. 2012. Prosodia valorativa: construcción de eventos y procesos en el discurso de la historia. Discurso \& Sociedad, 6 (2): 418-446. 
.2016. Appraisal framework and critical discourse studies: a joint approach to the study of historical memories from an intermodal perspective. International Journal of Language Studies, 10(2): 5-32. PÉREZ GALVÁN, Luis; OCHOA CERVANTES, Azucena. 2017. E1 aprendizaje servicio (APS) como estrategia para educar en ciudadanía. Alteridad, 12(2): 175-187.

TRAVER, Joan; MOLINER, Odet; SALES, Auxiliadora. 2019. Negociando el currículum: Aprendizaje-Servicio en la escuela incluida. Alteridad, Revista de Educación, 14.2.: 195-206.

URBINA-GARCÍA, Miguel Ángel. 2019. Methodological Strategies to Listen to Children's Voices: A Systematic Critical Review. Revista Colombiana de Educación, 77: 1-25.

VAN LEEUWEN, Theo. 2008. Discourse and practice. New Tools for critical discourse Analysis. Oxford: University Press.

VERGARA, Ana; PEÑA, Mónica; CHÁVEZ, Paulina; VERGARA, Enrique. 2015. Los niños como sujetos sociales: el aporte de los nuevos estudios sociales de la infancia y el análisis crítico del discurso. Psicoperspectivas, 14(1): 55-65.

WHITE, Peter. 2003. Beyond modality and hedging: A dialogic view of the language of intersubjective stance. Text, 23(2): 259-284.

WODAK, Ruth. 1999. Legitimizing Immigration Control: A discoursehistorical analysis. Discourse Studies 1.1: 83-119.

2011. La historia en construcción/La construcción de la historia. La "Wehrmacht alemana" en los recuerdos colectivos e individuales de Austria. Discurso \& Sociedad, 5 (1): 160-195.

Recebido em: 26/01/2020

Aprovado em: 06/10/2020 